\title{
Editorial
}

Psychopathology

\section{Parental Bonding and Dyadic Interaction: Source of Mental Health and Risk of Psychopathology}

\author{
Corinna Reck ${ }^{\mathrm{a}}$ Sabine C. Herpertz ${ }^{\mathrm{b}}$ \\ a Department of Psychology, Ludwig Maximilian University, Munich, and ${ }^{\mathrm{b}}$ Department of General Psychiatry, \\ Center for Psychosocial Medicine, University of Heidelberg, Heidelberg, Germany
}

A growing body of literature has focused on variables relevant for optimal cognitive, emotional, and behavioral development of children. One important factor accounting for this link is parenting and interaction behavior. Over the last 20 years, the concept of parental bonding in the postpartum period has come into focus. Bonding is described as the emotional tie from a mother to her newborn child [1] and includes behaviors such as proximity seeking, touch, contact, gaze, baby talk, positive expression, cuddling, and smiling [2] as well as cognitive-emotional variables such as positive feelings, emotional warmth, and affection [1]. Bonding develops gradually after birth, ensures protection and nurture of the newborn, is highly relevant for establishing the mother-infant relationship [3], and directly affects the way a parent interacts with the child. As a child's environment and socialization seem to play a crucial role in the development of his/her cognitive, emotional, and behavioral development, it can be assumed that satisfying interactional processes exerts a positive influence on a child's development. In the so-called mutual regulation model [4], interaction is regarded as a continuous moment-to-moment process, in which each partner adjusts to the behavior of the other [5]. The behavior of one partner during the interaction can be predicted by the

\section{KARGER}

E-Mail karger@karger.com

www.karger.com/psp behavior of the other in both positive and negative affective states. The ability of the caregiver-infant dyad to change in a flexible process between affective interactive 'matches' (coordinated behavior and affective states) and 'mismatches' (uncoordinated behavior and affective states) is of essential importance. The experience that negative affective states can successfully and reliably be transformed into positive states ('interactive repair') results in a sense of self-efficacy in infants and helps them to learn effective interactive strategies for affect regulation [4]. Studies identified these interactive processes as pivotal aspects of interactive regulation which exert influences on the socio-emotional and cognitive development of infants [6].

One factor which has been negatively associated with child development, bonding capacities, parenting, and interaction behavior is impaired mental health of the caregiver, especially if the mother is affected. Depression and anxiety disorders are among the most common psychiatric disorders and often affect women during the postpartum period. For Germany, prevalence rates of $6 \%$ for postpartum depression and $11 \%$ for postpartum anxiety disorders have been reported [7]. Maternal mental health is considered to affect child development in two ways: (1) a long-lasting negative impact of maternal men- 
tal health disorders on child development has been reported [8] and (2) an elevated risk of transmission for the offspring of parents with these diagnoses has been found $[9,10]$. Nevertheless, the status of research about risk factors for child development is that negative developmental outcomes do not solely depend on maternal psychiatric disorders but rather that the quality of interaction between mother and child is an important pathway for intergenerational transmission. Early life adversities in mothers have recently been discussed to be another severe risk factor for unfavorable child development, resulting in the intergenerational transmission of sequelae of trauma from mothers to offspring [11]. Most strikingly, an estimated $20-30 \%$ of abused children are likely to become abusive parents themselves.

To sum up, we can conclude that the quality of parenting behavior seems to be a risk factor for adverse child outcome. Interaction with a social partner plays an essential role in child development, and we assume that satisfying interactional processes exert a positive influence for child development. There are strong indications that the ability for supportive interaction with the child might be hampered in mothers with impaired bonding, negative parenting behavior, psychiatric disorders, or early life maltreatment in the own childhood.

This special issue is dedicated to the significant issue of the mutual influence of interpersonal experiences early in life and mental health later in life, which has also been focused on by several large funding initiatives dur- ing the last years (e.g. the National Institute of Health, NIH, or the Federal Ministry of Research and Education, BMBF, Germany). The main aim is to offer an updated survey of contemporary research on the impacts of successful bonding [Pena and Arias, pp. 217-227] as well as troubled rearing conditions [Brockington, pp. 247-260; Taubner et al., pp. 236-246; Bozicevic et al., pp. 228-235] that may be associated with the psychopathology of the mother [Fuchs et al., pp. 211-216; Reck et al., pp. 277284; Riva Crugnola et al., pp. 285-294; Müller et al., pp. 295-304] and/or early life maltreatment [Matas et al., pp. 201-210; Hillmann et al., pp. 261-268; Behrendt et al., pp. 269-276; Morelen et al., pp. 305-314]. The articles of this issue will shed new light on behavioral and biological mechanisms of healthy development as against sequelae of early life stress. We hope that important aspects for early prevention and mother-infant interventions will be derived from the special topics of the single papers. The findings suggest that mother-infant-centered intervention in the field of postpartum mental disorders, impaired bonding, and nonsupportive mother-child interaction should, besides the treatment of maternal mental health problems, focus on the improvement of the quality of interaction and the ability for interactive coordination and repair as well as on maternal interaction behavior. Addressing these issues in intervention and early prevention programs should have a positive effect on early development through the consolidation of a supportive motherchild interaction.

\section{References}

1 Klaus M, Kennell J, Klaus P: Bonding. New York, Addison-Wesley, 1995.

2 Reck C, Klier CM, Pabst K, et al: The German version of the Postpartum Bonding Instrument: psychometric properties and association with postpartum depression. Arch Womens Ment Health 2006;9:265-271.

3 Brockington I: Postpartum psychiatric disorders. Lancet 2004;363:303-310.

4 Tronick EZ: The Neurobehavioral and SocialEmotional Development of Infants and Children. The Norton Series on Interpersonal Neurobiology, New York, Norton \& Co, 2007.
5 Tronick EZ, Cohn JF: Infant-mother face-toface interaction: age and gender differences in coordination and the occurrence of miscoordination. Child Dev 1989;60:85-92.

6 Feldman R: Parent-infant synchrony: biological foundations and developmental outcomes. Curr Dir Psychol Sci 2007; 16:340345.

7 Reck C, Struben K, Backenstrass M, et al: Prevalence, onset and comorbidity of postpartum anxiety and depressive disorders. Acta Psychiatr Scand 2008;459-468.

8 Kingston D, Tough S: Prenatal and postnatal maternal mental health and school-age child development: a systematic review. Matern Child Health J 2014;18:1728-1741. $\checkmark 9$ Merikangas KR, Cui L, Heaton L, et al: Independence of familial transmission of mania and depression: results of the NIMH family study of affective spectrum disorders. Mol Psychiatry 2014;19:214-219.

10 Lebowitz ER, Leckman JF, Silverman WK, et al: Cross-generational influences on childhood anxiety disorders: pathways and mechanisms. J Neural Transm (Vienna) 2016, DOI: 10.1007/s00702-016-1565-y.

11 Widom CS: The cycle of violence. Science 1989;244:160-166. 